\title{
Utilização de Sargassum vulgare C. Agardh na adubação de Helianthus annuus L. (girassol)
}

\author{
Sheila Lorena Araujo Coelho', Sheila Vitória Resende ${ }^{2}$ \& José Marcos de Castro Nunes ${ }^{2}$ \\ 'Universidade do Estado da Bahia, Campus II, Herbário HUNEB, BR 110, Km 03. CEP: 41150-000. \\ 2Universidade Federal da Bahia, Instituto de Biologia, CEP: 40170-290, Salvador-BA
}

\section{Correspondência}

J.M. de Castro Nunes

e-mail: jmcnunes@ufba.br.

Recibido: 25 mayo 2017

Aceptado: 14 diciembre 2017

Publicado on-line: diciembre 2017

\begin{abstract}
Resumo
Este estudo propõe avaliar o potencial de Sargassum vulgare como fertilizante no crescimento do girassol. O delineamento experimental utilizado para análise do comprimento da parte aérea foi inteiramente casualizado em arranjo fatorial $5 \times 2 \times 7$ (concentrações de farinha desidratada de $S$. vulgare x concentrações de húmus de esterco bovino e caprino $x$ períodos de avaliação), com oito repetições e uma planta por repetição. Para análise área foliar e massa seca total o delineamento experimental utilizado foi inteiramente casualizado em arranjo fatorial $5 \times 2$ (concentrações de farinha desidratada de $S$. vulgare $\times$ concentrações de húmus), com quatro repetições e uma planta por repetição. Os resultados indicam que, apesar de ter havido um aumento do crescimento da parte aérea das plantas nos períodos avaliados, não houve o incremento deste crescimento ao utilizar a farinha desidratada de $S$. vulgare nas concentrações acima de 1\% na ausência do húmus.
\end{abstract}

Palavras-chave: Análise de crescimento, alga marinha, fertilizante.

\begin{abstract}
Seaweed Sargassum vulgare C. Agardh as fertilizer in the culture of the Helianthus annuus L. (sunflower)

The present study intends to evaluate the potential of Sargassum vulgare as fertilizer in the growth and development of the sunflower. A randomly delineation adopted was made by a factorial arrangement of $5 \times 2 \times 7$ (dehydrated $S$. vulgare flour concentrations $x$ humus concentrations of bovine and goat manure $x$ evaluation periods) with eight replications and one plant per replication. Leaf area and total dry mass were analyzed by randomly delineation adopted was by a factorial arrangement of $5 \times 2$ (dehydrated $S$. vulgare flour concentrations $x$ humus concentrations), with four replications and one plant per replication. The results indicate that although there has been an increase in plant height in the evaluation period, there was no increase in plant height using dehydrated $S$. vulgare flour concentrations above $1 \%$ in the absence of humus.
\end{abstract}

Key words: Fertilization, growth analysis, seaweed.

\section{Introdução}

A utilização de adubos orgânicos é um dos meios utilizados para introduzir nutrientes em diferentes culturas e, embora não constem relatos na literatura a respeito do uso de algas marinhas na fertilização de plantas de girassol, outras culturas como tomate, alface, coentro, mamão, salsa e cebolinha já foram fertilizadas por algas dos gêneros Ulva, Sargassum, Lithothamnium e Ascophyllum em diversas regiões do mundo (Dantas et al., 1998; Gestinari, 1999; Teixeira 
et al., 2004; Hafle et al., 2009; Koyama et al., 2012; N'Yeurt \& lese, 2014). Produtos à base de algumas espécies de algas usadas na forma seca ou em extratos têm sido comercializados como bioestimulantes em países da Europa e nos Estados Unidos. Entre estes, destacam-se Folical $E^{\circledR}$, Phycarine ${ }^{\circledR}$, Roots ${ }^{\circledR}$, Selecal ${ }^{\circledR}$ e Tonialg ${ }^{\circledR}$, sendo, em geral, utilizados com o objetivo de aumentar a resistência de plantas a doenças (Stadnik, 2003).

Dentre as algas marinhas encontradas no litoral brasileiro, o gênero Sargassum destaca-se com alta diversidade, como um componente importante da flora de regiões tropicais e subtropicais de ambos os hemisférios do globo (Paula, 1998; Nunes, 1999). A importância do gênero alcança enorme visibilidade e está entre as algas marinhas mais exploradas para fins comerciais. Segundo Bula-Meyer (2004), as espécies deste gênero têm sido utilizadas como fertilizantes nos cultivos de Arachis hypogaea L. (amendoim), Carica papaya L. (mamão), Cocos nucifera L. (coco) e Coffea arabica L. (café) na China. São excelentes fontes de elementos traços como o $\mathrm{Fe}, \mathrm{Co}, \mathrm{Mn}, \mathrm{B}$ e $\mathrm{Zn}$, além de possuir cerca de 60 a $70 \%$ de metano, favorecendo o uso desta alga na produção de biogás (Bula-Meyer, 2004).

No Brasil, apesar da riqueza da flora algológica, o uso de algas marinhas na agricultura é restrito à adubação de pequenos plantios localizados em regiões litorâneas (Santos et al., 2013). Segundo estes autores, grande quantidade das algas arribadas nas praias da costa brasileira e que poderiam ser utilizadas na agricultura, são incineradas ou enterradas pelas prefeituras locais com a finalidade de eliminar o mau cheiro advindo da sua decomposição.

O Helianthus annuus L. é uma oleaginosa anual que se adapta a diferentes condições edafoclimáticas, o que possibilita ser cultivado em todas as regiões do Brasil (Zobiole, et al. 2010). Em solos de determinadas regiões em que há restrição de nutrientes, o crescimento e desenvolvimento do girassol, bem como de outras espécies vegetais, podem ser afetados.

A importância econômica atribuída às algas marinhas indubitavelmente funciona como um fator que impulsiona sua exploração e/ou explotação em várias partes do globo. Além do Sargassum, outros gêneros de algas marinhas como, Ecklonia, Laminaria, Alaria, Nereocystis, Macrocystis, Ascophyllum, Fucus, Ahnfeltia, Lithothamnion, Ulva, Gracilaria e Turbinaria, vem sendo utilizados na agricultura em diferentes países do mundo (Abreu, 2005).

Há ainda poucas pesquisas que relatam a eficiência das algas marinhas como fertilizante em sua forma natural, seca ou úmida, e o presente estudo busca ampliar este campo do conhecimento

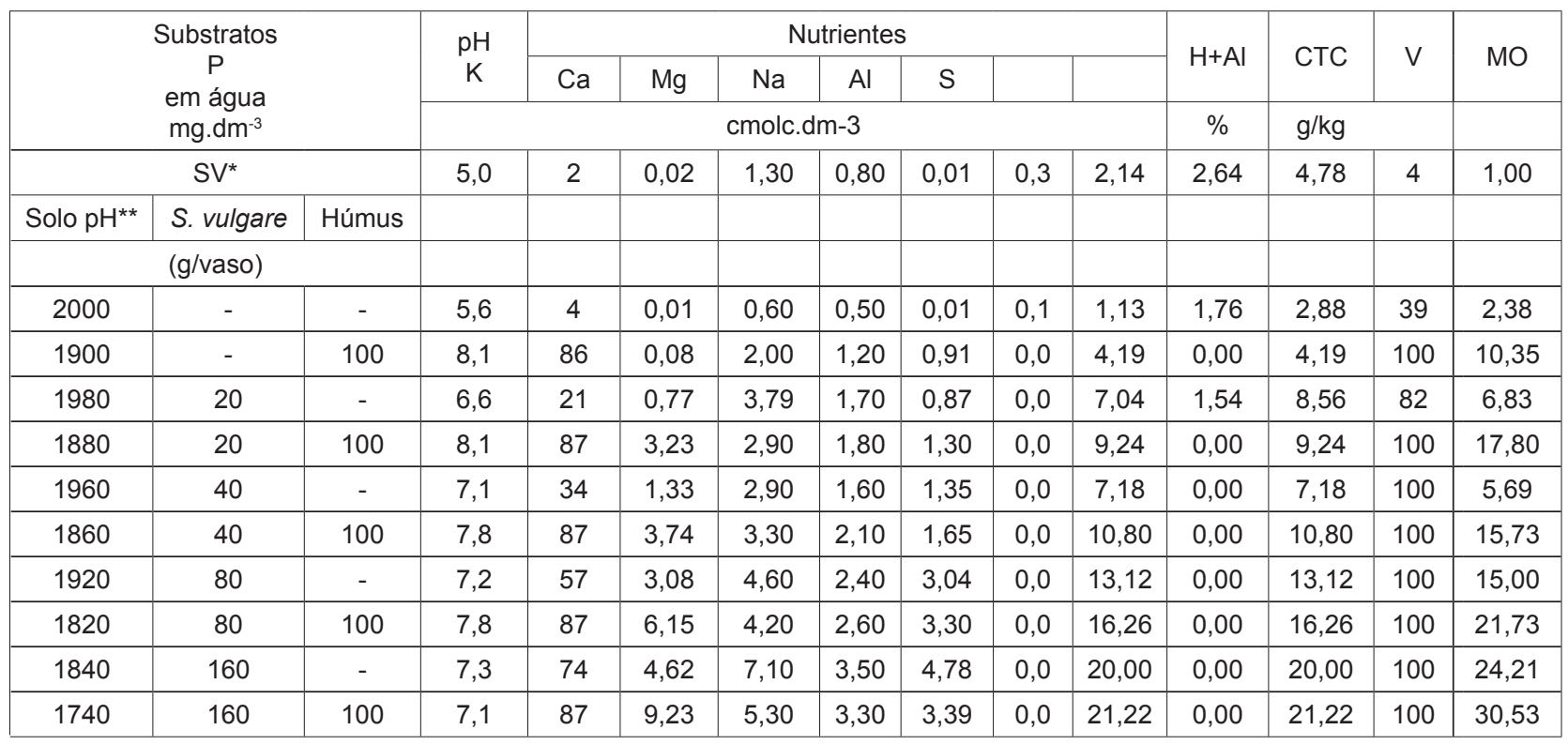

Tabela 1. Análise de fertilidade do solo dos tratamentos feitos no início do experimento no Laboratório de Solos e Nutrição de Plantas da Embrapa Mandioca e Fruticultura Tropical, Cruz das Almas - BA, 2010. Legenda: $\left({ }^{*}\right)-\mathrm{pH}$ não corrigido; ${ }^{* *} \mathrm{pH}$ corrigido; $\mathrm{H}+\mathrm{Al}$ - acidez potencial; CTC - capacidade de troca de cátions; V - saturação de bases; MO - matéria orgânica. Table 1. Soil fertility analysis of the treatments done at the beginning of the experiment at the Soil and Plant Nutrition Laboratory of the Embrapa Mandioca e Fruticultura Tropical, Cruz das Almas - BA, 2010. Legend: $\left(^{*}\right)$ - Corrected ph; $\left(^{\star *}\right)$ Uncorrected ph; $H+A l$ - potential acidity; CTC - cation exchange capacity; $V$ - base saturation; MO - organic matter. 
relativamente novo. Portanto, este trabalho propõe avaliar o potencial fertilizador da alga marinha Sargassum vulgare var. vulgare C. Agardh, com expressiva biomassa dentre as algas arribadas do litoral baiano.

\section{Material e métodos}

Os experimentos foram conduzidos em casa de vegetação e Laboratório de Solos da Universidade do Estado da Bahia (HUNEB), Campus II, Alagoinhas - BA.

As sementes (aquênios) de Helianthus annuus foram desinfestadas em solução de hipoclorito de sódio a $1 \%$ por cinco minutos (ISTA 1976) e semeadas em recipientes plásticos de $145 \mathrm{~mL}$. Após atingirem aproximadamente $6 \mathrm{~cm}$ de altura e com os cotilédones completamente expandidos, as plântulas foram transplantadas para os vasos contendo os substratos com adubo algáceo e húmus.

Para obtenção do adubo algáceo, exemplares de Sargassum vulgare foram coletados na Praia do Forte $\left(12^{\circ} 34^{\prime} 42.80^{\prime \prime} S 38^{\circ} 00^{\prime} 07.87^{\prime \prime} \mathrm{W}\right)$, no município de Mata de São João, Litoral Norte do Estado da Bahia, a cerca de $70 \mathrm{~km}$ de Salvador. Em laboratório, o material foi lavado em água corrente para remoção do sal presente nas frondes e posteriormente, submetido à secagem em estufa a $60^{\circ} \mathrm{C}$ por $96 \mathrm{~h}$. Após a completa desidratação, o material foi macerado em aparelho liquidificador até a obtenção de uma farinha.

O húmus utilizado foi adquirido comercialmente e composto de esterco puro de bovino e caprino.

O solo utilizado foi coletado a uma profundidade de 0-20 cm em uma área do Campus II da Universidade do Estado da Bahia, localizada sob as coordenadas $12^{\circ} 10^{\prime} 43^{\prime \prime} S$ e $38^{\circ} 24^{\prime} 44^{\prime \prime} \mathrm{W}$, classificado como Areias Quartzosas Álicas. Posteriormente o solo foi peneirado e levado à estufa à $60^{\circ} \mathrm{C}$ por $3 \mathrm{~h}$. Para a correção do $\mathrm{pH}$, foi realizada a calagem para $70 \%$ de saturação de bases (V\%), adicionando-se 0,7 g de calcário peneirado em malha de $500 \mathrm{~nm}$ em cada um dos vasos utilizados, seguindo as recomendações da Comissão Estadual de Fertilidade do Solo (1989). A incubação foi feita por um período de vinte dias.

As análises do solo e da fertilidade do substratoadubo foram realizadas pelo Laboratório de Solos e Nutrição de Plantas da Embrapa Mandioca e Fruticultura Tropical, Cruz das Almas - BA (Tab. 1) (Donagema et al., 2011).

No preparo do substrato contendo o adubo, foi adicionado $2 \mathrm{~kg}$ de solo com $\mathrm{pH}$ corrigido contendo farinha desidratada de $S$. vulgare nas concentrações $0,1,2,4$ e $8 \%(0,20,40,80$ e $160 \mathrm{~g} /$ vaso) e húmus nas concentrações 0 e $5 \%$ (0 e 100 $\mathrm{g} / \mathrm{vaso}$ ). Os vasos foram irrigados diariamente com $100 \mathrm{~mL}$ de água e no $20^{\circ}$ dia após a semeadura (DAS) houve a adição de $10 \mathrm{~mL}$ de solução nutritiva de Hoagland a $10 \%$ em cada vaso.

Aos $7,14,21,28,35,42$ e 49 DAS foram realizadas avaliações do comprimento da parte aérea, medido com régua graduada da base do caule até a última folha desenvolvida. No $50^{\circ}$ dia, período no qual teve início a fase reprodutiva, foram avaliadas a área foliar, a massa seca total e o teor de clorofila total.

A área foliar foi calculada segundo a metodologia proposta por Rawson e Constable (1980), utilizando a fórmula $A F=C . L .0,71, e m$ que, $\mathrm{C}=$ maior comprimento e $\mathrm{L}=$ maior largura. $\mathrm{A}$ área foliar por planta foi determinada pelo somatório da área foliar de cada folha. Os resultados foram expressos em $\mathrm{cm}^{2}$.

A matéria seca foi determinada após secagem em estufa a $65^{\circ} \mathrm{C}$ por $72 \mathrm{~h}$. Os resultados foram expressos em gramas de peso seco $(\mathrm{g})$.

0 delineamento experimental utilizado para análise do comprimento da parte áerea foi inteiramente casualizado em arranjo fatorial $5 \mathrm{x}$ $2 \times 7$ (concentrações de farinha desidratada de S. vulgare $\mathrm{x}$ concentrações de húmus $\mathrm{x}$ períodos de avaliação), com oito repetições e uma planta por repetição. Para análise área foliar e massa seca total o delineamento experimental utilizado foi inteiramente casualizado em arranjo fatorial 5 x 2 (concentrações de farinha desidratada de $S$. vulgare $\mathrm{x}$ concentrações de húmus), com quatro repetições e uma planta por repetição.

Os dados de comprimento da parte aérea foram submetidos a análise de variância utilizando o programa estatístico Sisvar (Ferreira 2011). Para o comprimento da parte aérea houve o ajuste das equações de regressão para descrever - comportamento de crescimento das plantas em função do tempo e para os parâmetros área foliar, massa seca total e teor de clorofila total as equações de regressão foram ajustadas em função do fator Sargassum. As médias foram comparadas pela análise de regressão e pelo teste de TukeyKramer a $5 \%$ de probabilidade.

\section{Resultados e discussão}

A análise da composição química nos talos de Sargassum vulgare var. vulgare indicou a presença de importantes componentes nutricionais (Tab. 1).

$\mathrm{Na}$ análise do comprimento da parte aérea houve interação significativa entre os fatores concentrações de farinha desidratada de $S$. vulgare, concentrações de húmus e períodos 

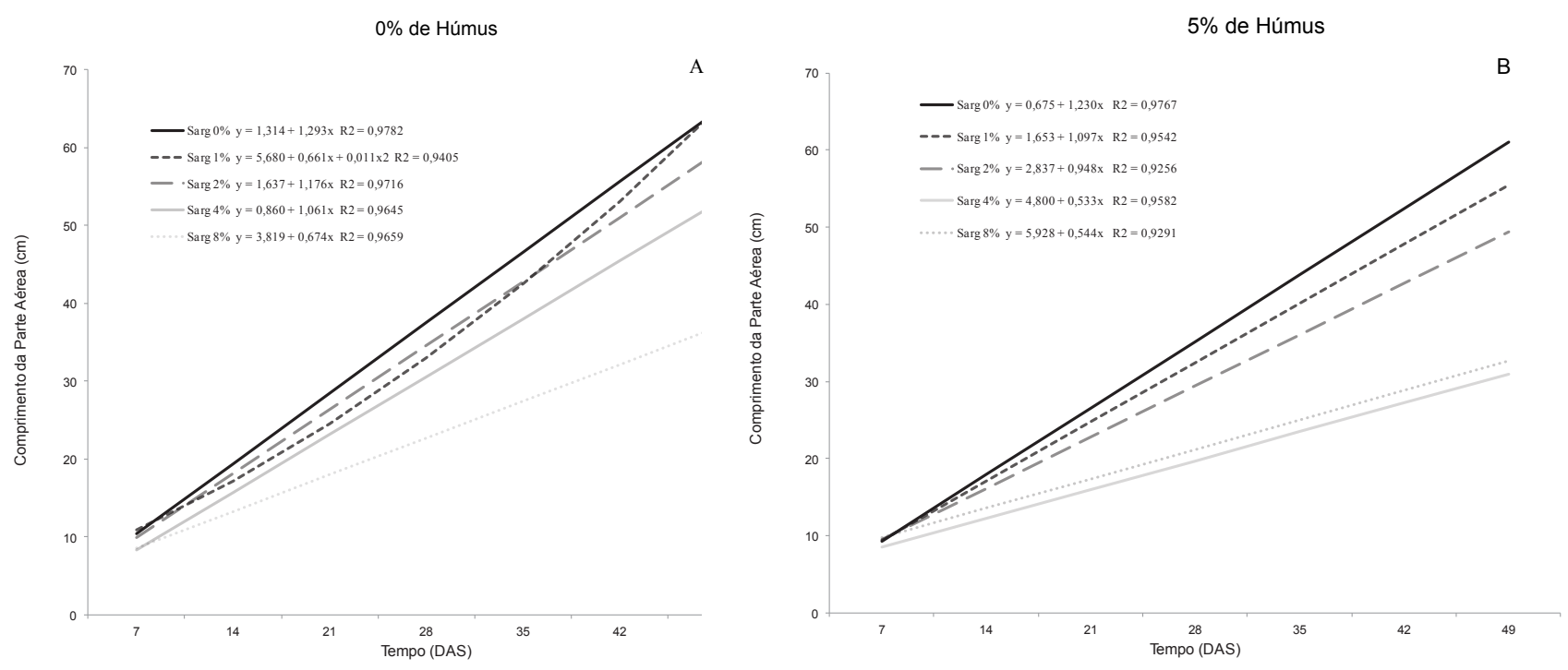

Figura 1. Variação do comprimento da parte aérea (cm) de Helianthus annuus em cada concentração (0, 1, 2, 4 e 8\%) de farinha desidratada de Sargassum vulgare utilizada e concentração de $0 \%$ de húmus $(\mathbf{A})$ e $5 \%$ de húmus (B), em função dos diferentes períodos de avaliação (7, 14, 21, 28, 35, 42 e 49 DAS). $n=8$. Figure 1. Variation in shoot length (cm) of Helianthus annuus in each concentration (0, 1, 2, 4 and 8\%) of flour dried Sargassum vulgare used and $0 \%$ concentration of humus (A) and 5\% humus (B), according to the different collection periods (7, 14, 21, 28, 35, 42 and 49 Dias após a semeadura- DAS). $n=8$.

de avaliação. De forma geral, o aumento da concentração de farinha desidratada de $S$. vulgare e de húmus proporcionou um aumento do comprimento da parte aérea de girassol ao longo do período de avaliação, tendo um incremento diário no comprimento da parte aérea de $0,984 \mathrm{~cm}$ (fig. 1).

Os resultados demonstram que, apesar de ter havido um aumento do crescimento da parte aérea das plantas nos períodos avaliados, não houve o incremento deste crescimento ao utilizar a farinha desidratada de $S$. vulgare nas concentrações a partir de $1 \%$ na ausência do húmus (fig. 1A). Resultado semelhante foi observado por Gestinari (1999) ao estudar o efeito das macroalgas marinhas Ulva e Sargassum em Allium schoenoprasum L. (cebolinha), Raphanus sativus L. (rabanete) e Petroselinum crispum (Miller) Hill (salsa), não encontrou efeito positivo no comprimento de parte aérea em nenhuma das culturas analisadas. Gestinari (1999) observou um decréscimo no comprimento da parte aérea das plantas à medida que houve o aumento nas concentrações dos fertilizantes.

Dantas et al. (1998) observaram um melhor desenvolvimento das folhas de Lactuca sativa $L$. (alface) e Coriandrum sativum L. (coentro) quando foi adicionado à areia quartzosa marinha $25 \%$ de farinha desidratada de $S$. vulgare superando 0 tratamento à base de $25 \%$ de esterco bovino.

Hafle et al. (2009), observaram efeito positivo no comprimento da parte aérea de mudas de mamoeiro ao utilizar pó da algas marinhas do gênero Lithothamnium. Os melhores resultados encontrados por esses autores ocorreu nos tratamentos em que as doses de Lithothamnium interagiram com o Bokashi, um fertilizante orgânico baseado em solução líquida de bactérias anaeróbicas e fermentos do ácido lático (Hafle et al., 2009). No estudo atual, os resultados para comprimento da parte aérea em girassol demonstram que o húmus não interagiu positivamente com a alga marinha Sargassum. $\mathrm{Na}$ presença do húmus pode-se observar um decréscimo significativo no comprimento da parte aérea das plantas de girassol com o aumento da concentração da farinha desidratada de S. vulgare, o que pode estar relacionado a uma maior retenção de sais no substrato de cultivo (fig. 1B).

Ayers \& Westcot (1987) apontam que o aumento da pressão osmótica do solo ocasionado pelo aumento da concentração de íons, atua de forma negativa sobre os processos fisiológicos, reduzindo a absorção de água pelas raízes, inibindo a atividade meristemática e o alongamento celular promovendo a redução do crescimento das plantas.

Em relação à área foliar não houve interação significativa entre os fatores concentrações de farinha desidratada de $S$. vulgare e concentrações de húmus. De acordo com o modelo de regressão para esta variável, houve um comportamento linear decrescente em função do aumento das concentrações de S. vulgare (fig. 2). Na presença 


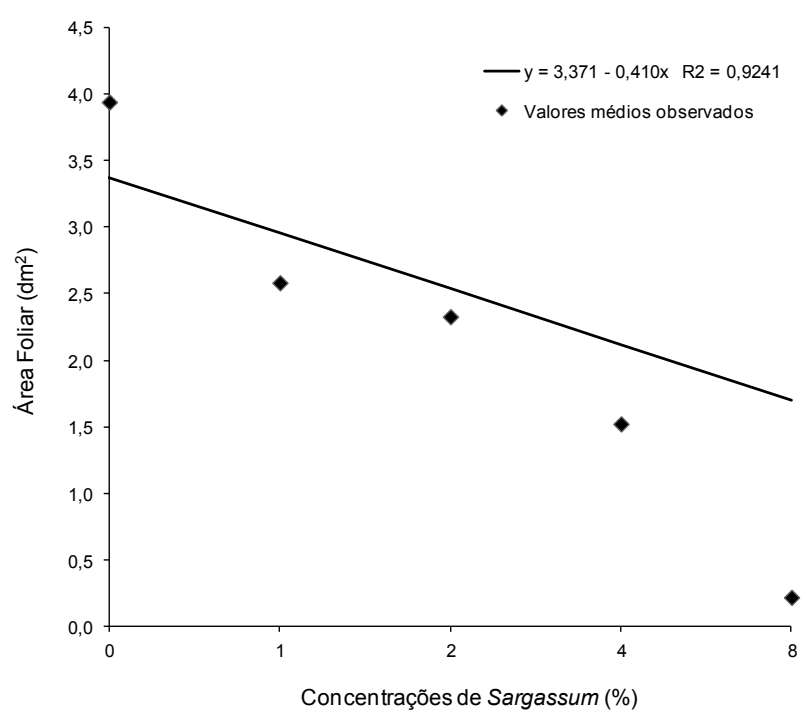

Figura 2. Área foliar $\left(\mathrm{cm}^{2}\right)$ de girassol em função das concentrações de farinha desidratada de Sargassum vulgare aplicadas. $\mathrm{n}=4$. Figure 2. Leaf area $\left(\mathrm{cm}^{2}\right)$ of sunflower as a function of the concentration of flour dried Sargassum vulgare applied. $n=4$.

da farinha desidratada de $S$. vulgare foi observado um decréscimo significativo de $0,417 \mathrm{~cm}^{2}$ da área foliar com o aumento das concentrações A maior média foi observada no tratamento controle, com $3,940 \mathrm{~cm}^{2}$.

Com relação à massa seca total não houve interação significativa entre os fatores concentrações de farinha desidratada de $S$. vulgare e concentrações de húmus.

Similar ao que foi observado para área foliar, o modelo de regressão mostra um comportamento linear decrescente em função do aumento das concentrações de $S$. vulgare (fig. 3). Esses resultados divergem daqueles observados por Dantas et al. (1998) no qual a massa seca das folhas de alface e coentro foi maior quando crescidas na presença de $25 \%$ de $S$. vulgare em areia quartzosa marinha.

\section{Conclusão}

A utilização do Sargassum vulgare como fertilizante na concentração a partir de 1\%, não é recomendada para o crescimento de Helianthus annuus por um período de 49 dias após semeadura.

\section{Agradecimentos}

À Fundação de Amparo á Pesquisa do Estado da Bahia (FAPESB) e ao Conselho Nacional de Desenvolvimento Científico e Tecnológico (CNPq) pela concessão da bolsa de iniciação científica. J.M.C. Nunes agradece ao CNPq pela Bolsa Produtividade.

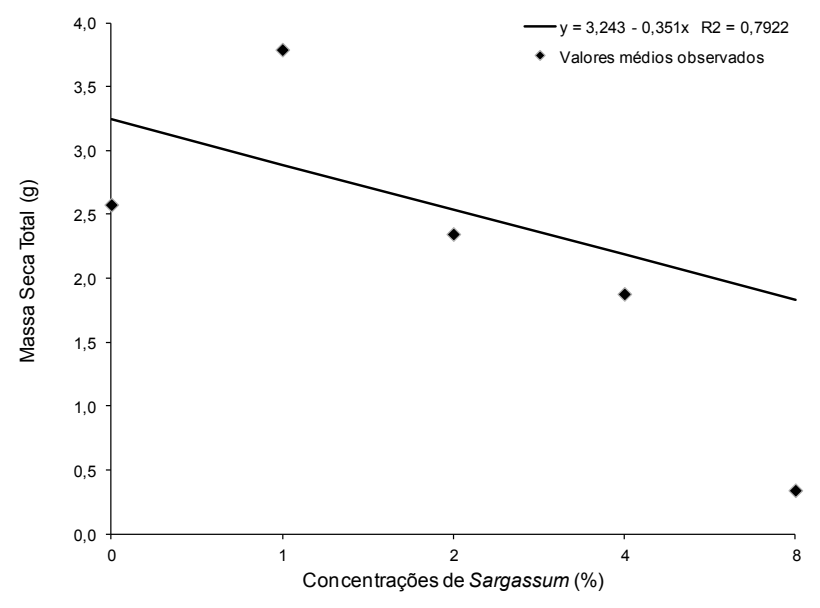

Figura 3. Massa seca total (g) Helianthus annuus em função das concentrações de farinha desidratada de Sargassum vulgare aplicadas. $\mathrm{n}=4$. Figure 3. Total dry weight $(\mathrm{g})$ of Helianthus annuus depending on the concentration of flour dried Sargassum vulgare applied. $n=4$.

\section{Referências}

Abreu, G. F. (2005). Bioprospecção de macroalgas marinhas e plantas aquáticas para o controle da antracnose (Colletotrichum lindemuthianum) do feijoeiro (Phaseolus vulgaris L.). Dissertação (Mestrado em Recursos Genéticos Vegetais), Universidade Federal de Santa Catarina, 80p.

Ayers, R.S. \& Westcot, D.W. (1987). A qualidade da água na agricultura. Roma: FAO. (FAO. Estudo FAO Irrigação e Drenagem, 29). 218p.

Bula-Meyer, G. (2004). Las macroalgas marinas em la agronomia y el uso potencial del Sargassum flotante em la producción de fertilizantes en el Archipiélago de San Andrés y Providencia, Colombia. Intropica, 1, 91103.

Comissão Estadual de Fertilidade do Solo- CEFS (1989). Manual de adubação e calagem para o Estado da Bahia. CEPLAC/EMBRAPA/SEAGRI, Salvador. 173p.

Dantas, N.P., Joventino, F.P. \& Santos, J.H.R. (1998). Efeitos de variadas concentrações de Sargassum vulgare $\mathrm{C}$. Agardh no crescimento de alface e coentro. Arquivos de Ciência do Mar, 31, 41-46.

Donagema, G.K., De Campos, D.V.B., Calderano, S. B., Teixeira, W. G. \& Viana, J. H. M. (2011). Manual de métodos de análise de solo. EMBRAPA. 230p.

Ferreira, D.F. (2011). Sisvar: a computer statistical analysis system. Ciência e Agrotecnologia, 35, 10391042.

Gestinari, L.M.S. (1999). Macroalgas marinhas como bioestimulantes na olericultura. Dissertação (Mestrado em Biotecnologia Vegetal). Universidade Federal do Rio de Janeiro, Rio de Janeiro, 1999. 196p.

Hafle, O.M., Santos, V.A., Ramos, J.D., Cruz, M.C.M. \& Melo, O.C. (2009). Produção de mudas de mamoeiro utilizando bokashi e Lithothamnium. Revista Brasileira de Fruticultura, 31(1), 245-251.

International Seed Testing Association. 1976. Seed 
health testing. Seed Science and Technology, 4, 3-49. Koyama, R., Bettoni, M.M., Roder, C., Assis, A.M., Roberto, S.R. \& Mógor, A.F. (2012). Extrato de alga Ascophyllum nodosum (L.) Le Jolis no desenvolvimento vegetativo e na produção do tomateiro. Revista de Ciências Agrárias, 55(4), 282-287.

Nunes, J.M.C. (1999). Phaeophyta da Região Metropolitana de Salvador, Bahia, Brasil. São Paulo: USP. 271f. Dissertação (Mestrado em em Ciências Biológicas - Botânica), Universidade de São Paulo, São Paulo, 1999.

N'yeurt, A.R. \& Viliamu, I. (2014). The proliferating brown alga Sargassum polycystum in Tuvalu, South Pacific: assessment of the bloom and applications to local agriculture and sustainable energy. Journal Applied Phycology, 26, 1-9.

Paula, E.J. (1998). O gênero Sargassum C. Agardh (Phaeophyta, Fucales) no litoral do Estado de São Paulo, Brasil. Boletim de Botânica, 10, 65-118.

Rawson, H.M. \& Constable, G.A. (1980). Carbon production of sunflowers cultivars in field and controlled environments. I. Photosynthesis and transpiration of leaves, stems and heads. Australian Journal of Plant
Physiology, 2(5), 555-573.

Rizzini, C.T. \& Mors, W.B. (1995). Botânica econômica brasileira. 2 ed. Âmbito cultural: Rio de Janeiro. 248p. Santos, G.N., Nascimento, O.S., Pedreira, F.A., Rios, G.I., Vasconcelos, J.N.C. \& Nunes, J.M.C. (2013). Análise quali-quantitativa das algas arribadas do Norte do Estado da Bahia, Brasil. Acta Bot. Malacitana, 38, 13-24.

Stadnik, M.J. Bettiol, W. \& Saito, M.L. (2003). Bioprospecting for plant and fungus extracts with systemic effect to control the cucumber powdery mildew. Journal of Plant Disease and Protection, 110, 383-393.

Teixeira, N.T., Paula, E.L., Fávari, D.B., Almeida, F. \& Guarnieri, V. (2004). Adubação orgânica e organomineral e algas marinhas na produção de alface. Revista Ecossistema, 29(2), 19-22.

Ungaro, M.R.G. (2000). Cultura do girassol. Boletim técnico, 188. Instituto Agronômico: Campinas. 36p.

Zobiole, L.H.S., Castro, C., Oliveira, F.A. \& Oliveira Junior, A. (2010). Marcha de absorção de macronutrientes na cultura do girassol. Revista Brasileira de Ciência do Solo, 34, 425-433. 